\title{
Polymer Microring Resonator Directly Patterned by Multilevel-Nanoimprint: Integration into a Biosensor System with a Miniaturized Microfluidic System
}

Rene Landgraf ${ }^{1,2},+49351$ 8823-487, rene.landgraf@ipms.fraunhofer.de Robert Kirchner 2,1 , +49 351 463-36414, robert.kirchner@tu-dresden.de Andreas Finn ${ }^{2,1}$, +49 351 463-36414, andreas.finn@mailbox.tu-dresden.de Susann Arndt ${ }^{2}$, 49351 463-32653, susann.arndt@mailbox.tu-dresden.de Toni Haugwitz ${ }^{3}$, +49 351 463-35884, toni.haugwitz@tu-dresden.de Frank Deicke1, +49 351 8823-385, frank.deicke@ipms.fraunhofer.de Wolf-Joachim Fischer ${ }^{2,1}$ +49 351 463-36336, wolf-joachim.fischer@tu-dresden.de

1 Fraunhofer-Institute for Photonic Microsystems, Maria-Reiche-Str. 2, 01109 Dresden, Germany

2 Technische Universität Dresden, Inst. for Semiconductors and Microsystems, 01062 Dresden, Germany

${ }^{3}$ Technische Universität Dresden, Communications Laboratory, 01062 Dresden, Germany

\begin{abstract}
With optical biosensors based on planar optical waveguides, much progress was achieved in the last decade. Due to their high sensitivity, small size and good ability for integration, waveguides of microring resonator shape were identified to be especially suited for biosensor applications. Biosensing with microring resonators is typically carried out with reliable but bulky lab tools. Sensors are typically not integrated in portable sensor systems with a microfluidic system, laser source, detector as well as grating couplers. For point-of-care testing (POCT), these peripheral components are essential for an independent system usage. The biosensor system proposed in this work is based on a sensor chip with functional, polymeric microring resonators. With multilevel grating couplers, a miniaturized microfluidic system and cost-efficient UV-based nanoimprint (UV-NIL) manufacturing technology, the biosensor system has a high potential for further miniaturization and the use for POCT. The main focus in this work is the integration of a microfluidic system that allows the supply with analyte solutions and the processing of automated biosensor experiments. The influence of the microfluidic system on the sensing performance is discussed.
\end{abstract}

\section{Introduction}

Because of their high sensitivity and ease of integration, optical sensors based on integrated waveguides have been strongly put forward in the last decade [1]. Especially microring resonator shaped waveguides have been of interest, due to their independence to the waveguide interaction length and their advantageous transmission curve with steep slopes for high sensitivity sensing (Fig. 1).

a)

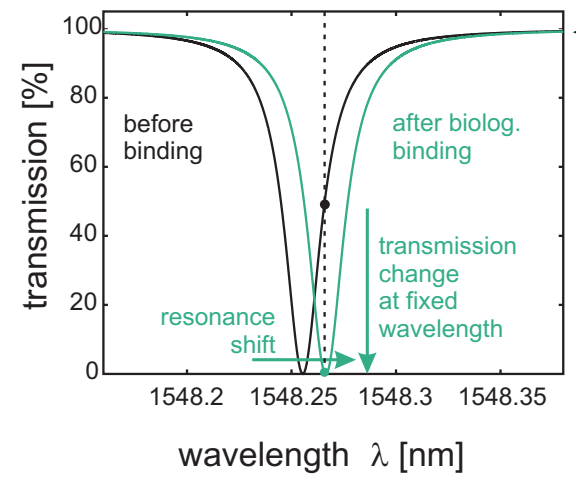

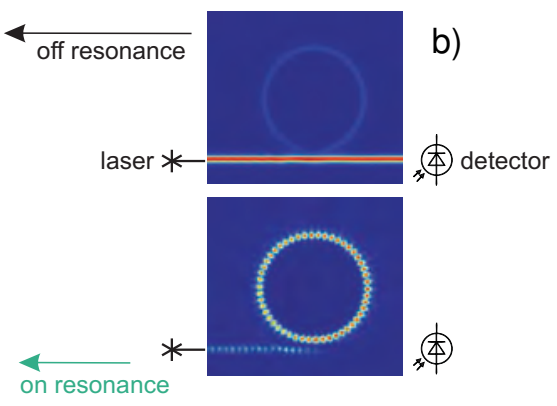

on resonance

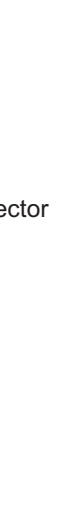

Fig. 1: Transmission curve for a biosensor experiment before and after binding of biological molecules for a typical polymer-on- $\mathrm{SiO}_{2}$ waveguide (a) calculated after Yariv [2] assuming critical coupling, and power intensities of a non-resonant (top) and resonant (bottom) microring resonator (b).

Due to the small microring resonator footprint with diameters of typically some $10 \mu \mathrm{m}$, the integration in arrays is possible, allowing parallel measurements with multiple microring resonators [3]. Since the active ring surface area, that needs to be covered with biological analyte is small, the amount of analyte can be further reduced. The sensitivity for biosensing with microring resonators depends particularly on the quality factor of the microring, which can be increased by design and manufacturing optimization [4-6]. Ultra-low limits of detection of as small as $1.5 \mathrm{pg} / \mathrm{mm}^{2}$ were extrapolated from measurements with planar silicon microring resonators [7], being equal to state-of-the-art surface plasmon resonance based 
biosensing. For optical microresonators with a 3-D toroidal shape, even single molecule detection was achieved [8]. For this type of optical microresonator, due to the free-standing toroidal shape, in- and outcoupling need to be carried out manually by tapered fibers. This is impossible for a portable POCT device that is operated by layman personal. A less sensitive design with planar microring resonators needs to be used. Typically, microring resonators for biosensing are made out of silicon with silicon-oninsulator technology [3, 7]. They are usually manufactured with high-resolution lithography and dry etching, which is costly and time-consuming. If polymer materials are used as waveguides, nanoimprint lithography (NIL) can be used for a potentially large volume production of low-cost biosensor chips. NIL is a direct patterning method for polymer micro and nano systems [9, 10] and allows the cost-efficient manufacturing of structures in the sub-300 nm range with aspect ratios of up to 10:1. UV-assisted NIL (UV-NIL) as used in this work, compared to thermal based NIL (T-NIL), offers an easier processing by using low-viscous imprint materials and the ability to add functional monomers for surface functional groups $[11,12]$.

For POCT with portable waveguide biosensing devices, peripheral parts of the system for aqueous solution supply and laser in- and outcoupling, play an important role for the independent operation of the system. One of the most critical points of the optical signal path is the light guiding to and from the optical chip. The planar optical waveguides on the chip have smaller mode diameters than the single-mode fibers used for light guiding to the periphery. Grating couplers enable coupling perpendicular to the chip surface, which improves the design flexibility, compared to butt- or prism-coupling. Grating couplers provide a large positioning tolerance by maintaining a high efficiency [13].

Most biosensors measure the analyte in an aqueous sample solution during the sensing experiment and therefore need a fluidic system. Industrially used fluidic systems are designed to be robust and controllable what makes them large and difficult to integrate. Many integrated approaches on the other hand are miniaturized, but only for short-term and low-pressure use. An example for a commercially available solution is the SmartFlow fluidic system [14], which is a stand-alone fluidic system that provides profiled flow rates. However, it is not miniaturized and difficult to integrate in an automated biosensor system. Further fluidic solutions are the systems of the companies GeSiM [15] and Syrris [16], partially used for automated analytical applications. In another study, LEGO ${ }^{\circledR}$ Mindstorms ${ }^{\circledR}$ NXT components are used to create a fluidic system [7]. All these systems are usually adapted for specific applications but are rather large or mainly manually controlled. We have developed a fluidic system that is miniaturized and robust, and allows the automation of entire biosensing experiments. For an immunoassay type of biosensing, a sequence of several process steps with sample, antibody and buffer solutions is necessary. An exemplary sensing experiment cycle is shown in Fig. 2. A first washing step of the sensor surface with buffer solution is followed by injection of a receptor solution. The receptors bind or adsorb on the sensor surface. Another washing step with buffer solution rinses away unbound receptors. The analyte molecules then bind to the receptors. To covalently bind receptors onto the surface, functional monomers are embedded within the waveguide polymer [12] or a preceding surface functionalization step of the optical microring resonator biosensors is carried out [17].

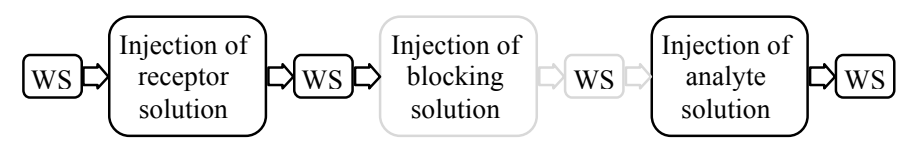

Fig. 2: Process flow of a typical biosensing experiment that can be carried out in a fully automated mode, including several washing steps (WS) with buffer solution, and an optional blocking step to improve selectivity.

In this work, we propose a biosensor system with multilevel grating couplers and a miniaturized microfluidic system. UV-NIL is used as a cost-efficient manufacturing technology. The main focus in this work is the integration of a microfluidic system that allows the supply with analyte solutions, and the processing of automated biosensor experiments. The influence of the microfluidic system on the sensing performance is discussed.

\section{Polymer Microring Resonator and Multilevel Grating Couplers}

Microring resonators consist of optical waveguides and have a characteristic transmission spectrum with resonance maxima or minima, depending on the setup with or without a drop waveguide [9]. The transmission spectrum shifts with surrounding media changes, i. e., the binding of biomolecules in the case of biological sensing (Figs. 1,3). 
a)

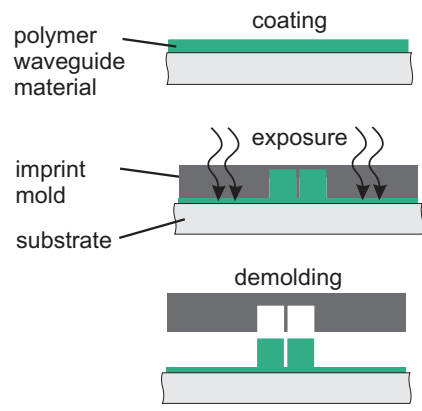

imprint

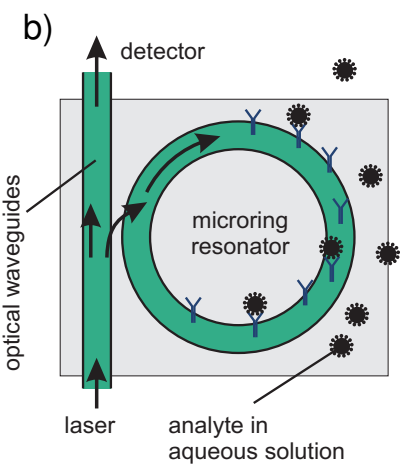

c)

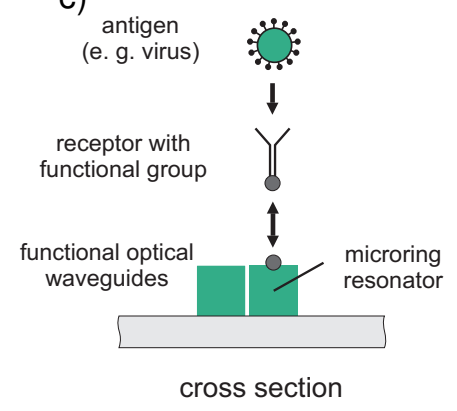

Fig. 3: Basic principle of affinity biosensing with polymer microring resonators: Structuring by UV-assisted nanoimprint lithography (a), biosensing by measuring of the transmission change during binding of the analyte (b) and specific binding of the analyte (antigen) to receptors that are covalently immobilized onto functional polymer waveguides (c).

Polymer waveguides need to have good mode confinement, which has to be considered during the design process. This is particularly important for microring resonators, where a decrease in the waveguide loss means a better detection limit. The optical design challenge, when applying NIL-manufacturing, is the limited aspect ratio and the use of low refractive index (RI) polymers, e. g., acrylates, especially for UV$\mathrm{NIL}$. The waveguides in this work were designed by analytical calculations using an adapted effective refractive index method [18] and finite elements simulations (FEM).

Fig. 4 shows a mode diagram, calculated with the adapted effective refractive index method [18], to determine suitable waveguide geometries and waveguide polymers. This diagram can also be displayed for a different substrate material. The area between the fundamental mode $(00)$ and the first modes in $x$ direction (10) and $y$-direction (01) represents the operating range for single mode propagation of a straight waveguide. LL displays the smallest waveguide and refractive index combination, where the coupling condition between two waveguides is fulfilled. This is necessary for the function of microring resonators, where modes couple between the bus and ring waveguides. UL shows the cut-off for the undesired higher modes during coupling. The colored area in the mode diagram between LL and UL defines the desired operating range including the fulfilled approximated coupling condition.

To connect the microring resonator element to a source and a detector, grating couplers are used. Thereby, shallow gratings in a waveguide (Fig. 5, (1)) ensure the mode size conversion from an optical fiber in perpendicular chip direction. Further, adiabatic tapers (Fig. 5, (2)) convert the mode in lateral direction. This setup facilitates the incoupling of the laser and outcoupling to the detector and thus enables the potential use as exchangeable single-use sensor chips.

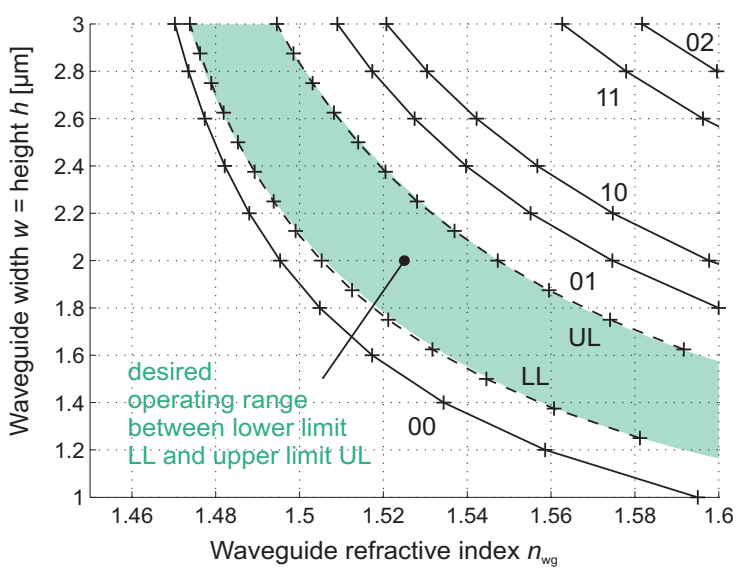

Fig. 4: Mode diagram for waveguides with a quadratic cross-section (parameters: $\mathrm{SiO}_{2}$ undercladding layer; at wavelength $\lambda=1550 \mathrm{~nm}$; TE polarization) showing the desired operating range.

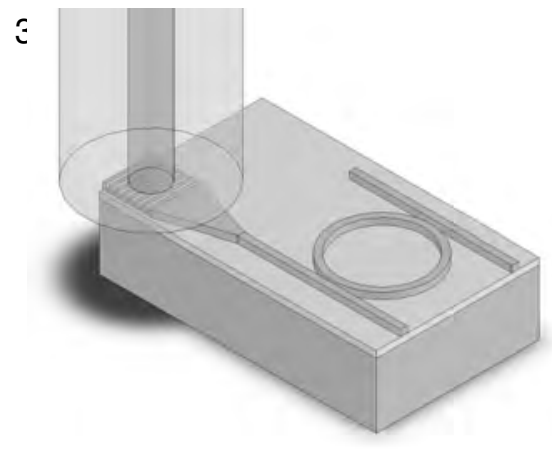

Fig. 5: Concept using grating couplers (1) and adiabatic tapers (2) for coupling from optical single mode fibers (3) to planar microring resonators (4). 


\section{Residual layer free and Multilevel UV-Nanoimprint Manufacturing}

A functional monomer system based on acrylates was developed [12]. The concentration of the functional monomer was altered to obtain different concentrations of surface-functional groups. After structuring by UV-NIL, hydroxyl groups should be accessible for covalent receptor immobilization without post-imprint treatment. Specific covalent binding of small molecules could be shown (Fig. 6). To avoid the NIL inherent polymer residual layer, hybrid nanoimprint molds were used. Chromium layers are used to block the light in unstructured areas, and thus prevent the underlying residual layer from cross-linking. Freestanding polymer structures were obtained on opaque substrates (Fig. 7).

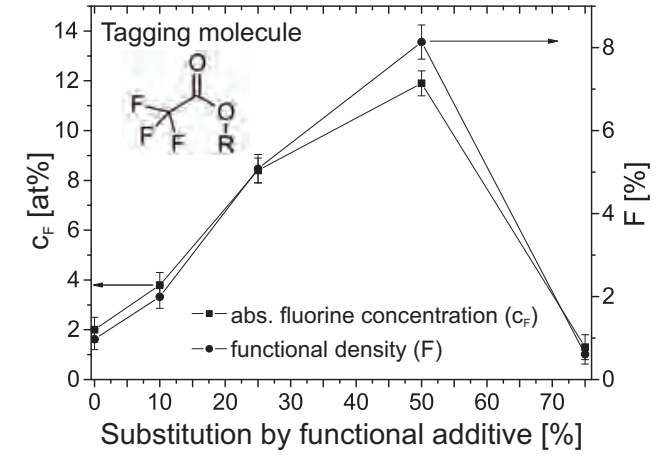

Fig. 6: Proof of specific binding of fluorinated tags onto hydroxyl terminated surfaces for different amounts of the functional additive.

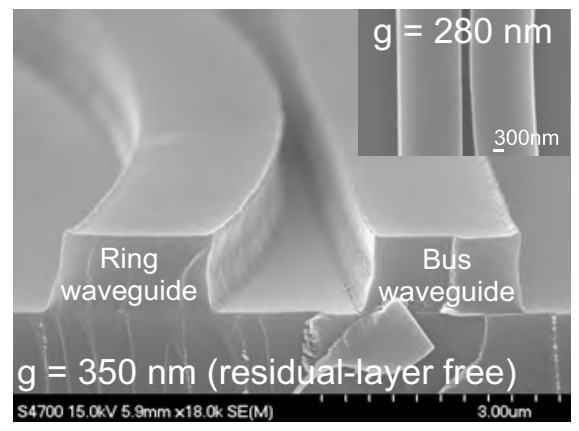

Fig. 7: Microring resonator with a gap $g=280 \mathrm{~nm}$ (inset) and a residual layer free microring resonator with $g=350 \mathrm{~nm}$.

The optical grating couplers are patterned in the same fabrication step as the microring resonator structures using UV-NIL. For this purpose, a special multilayer mold is used. For the production of these molds, a fabrication method was proposed [19], which ensures the necessary in-plane and out-of-plane resolution needed. Fig. 8 shows realized microring resonator structures with different height levels for the ring and the input waveguide.
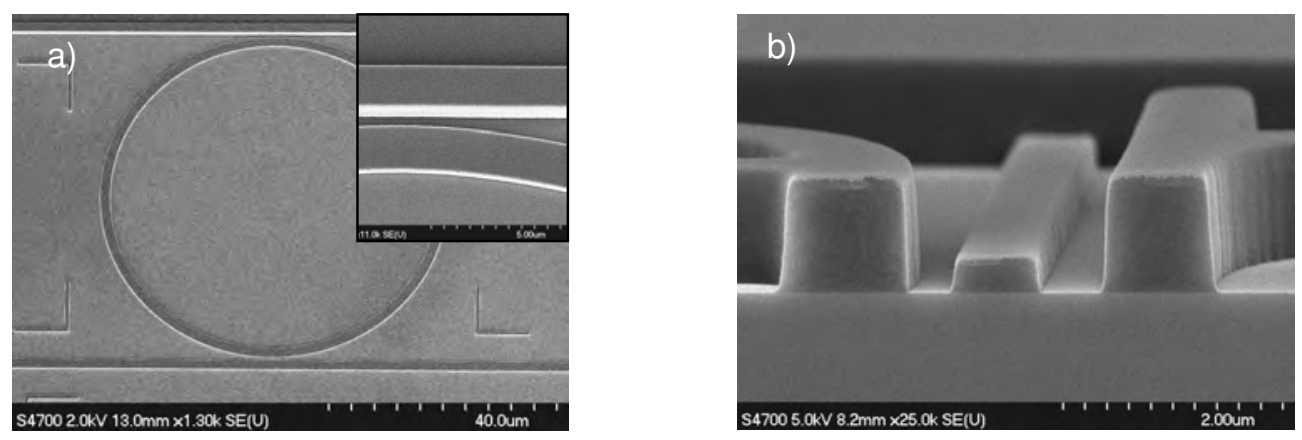

Fig. 8: Scanning electron micrographs of residual layer free polymer replication of multilayer NIL-molds showing a microring resonator, having a different height than the input-guide (top of the picture) (a) and a cross-section of a multilevel polymer structure (b).

\section{Biosensor System with Integrated Microfluidic System}

The here proposed biosensor system is based on a sensor chip with functional microring resonators for covalent binding of receptors. An optical/electronic part and a fluidic part are combined (Fig. 9). The aim of the fluidic system is to provide a miniaturized system for use within the optical biosensor system.

The fluidic system (Fig. 9) is based on a commercially available membrane micropump with inlet and outlet valves as well as magnetic microvalves for switching between the respective sample and other liquids. A fluidic board with fluidic channels was designed and is used for the mounting of the micro valves. With the multiple implemented connections, e. g., a receptor, sample and buffer solution can be accessed. This way, an entire measurement cycle for an immunoassay-like biodetection method (process flow shown in Fig. 2) can be processed fully automatedly. A valuable feature of the system is the swit- 
ching ability between an injection mode (Fig. 9, A), which allows the input of the different liquids, and a cycle mode. The cycle mode (Fig. 9, B) allows using smaller sample and test liquid volumes. The lab setup of the fluidic system is shown in Fig. 10. The fluidic system is controlled with standard lab control software Labview $^{\circledR}$. The flow rate can be adjusted and alters the switching frequency of the pump valves. If only very small volumes of a sample are at disposal, the system can be adapted (Fig. 11) so that the analyte consumption is reduced by shorter tubes and an alternating forward-backward flow direction. If steps with additional fluids are needed in a sensing experiment, the fluidic board can be easily extended with more valves.

Apart from refractive index changes in the surrounding media, microring resonator sensors are also sensitive to mechanical vibrations, e. g., caused by pumps and valves, as well as the pulsation of a fluid flowing over the microring waveguides. Both influences will cause small changes to the shape of the ring and thus will alter its resonant wavelengths. Another influencing factor is the temperature, mainly of the sample flowing over the sensor surface.

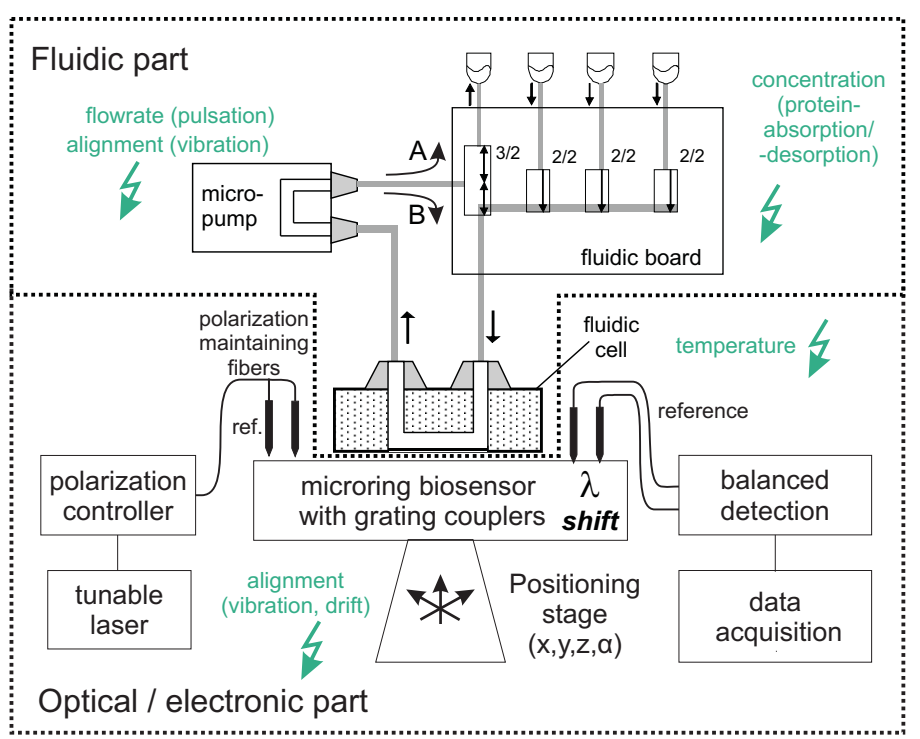

Fig. 9: Schematic overview of the optical biosensor system. Sensing is influenced by cross-sensitivities to changes in the alignment positions, flow rate, microring temperature and the absorption/desorption of matrix molecules (e. g. proteins) of the sensing solution.

For the fluidic system, the materials ethylenetetrafluoroethylene (ETFE, fittings), polydimethylsiloxane (PDMS, flow-cell), polyetheretherketone (PEEK, fluidic board and connectors) and polytetrafluoroethylene (PTFE, tubes) were used, because of their high chemical stability and relatively low surface energy and high biocompatibility. As a flow cell, a PDMS structure is used and is positioned on the sensor surface. PDMS was molded from a silicon master by replica molding. The connecting tubes were embedded in the same step. Drilling or punching in PDMS showed rough hole forming and was not used. ETFE, PTFE and PEEK can be cleaned using many solvents and weak bases and acids. Therefore, small residues of analyte and test solutions can be removed before the next experiment. In cases with stringent conditions for contaminations from previous experiments, an alternative setup with forward-backwards flow (Fig. 11, C) reduces the number of components in contact with the analyte solution.

\section{Influence of the Microfluidic System to Optical Biosensing}

We examined the influence of vibration, flow rate and binding of proteins and discuss the influence to the optical biosensing system. The suggested evaluation procedures can be applied as tests during microfluidic system development. 


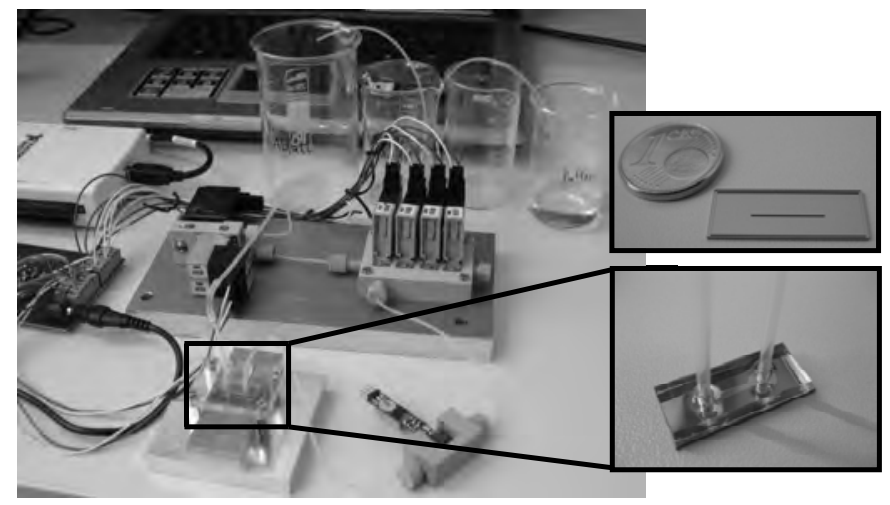

Fig. 10: Lab setup of the separated fluidic system during evaluation with micropump (1), flow cell (2) and connecting board with valves (3) as well as temperature sensor (4) and sensor / flow cell stage (5). The close-up pictures on the right side show a single channel silicon master (6) and a PDMS flow cell (2).

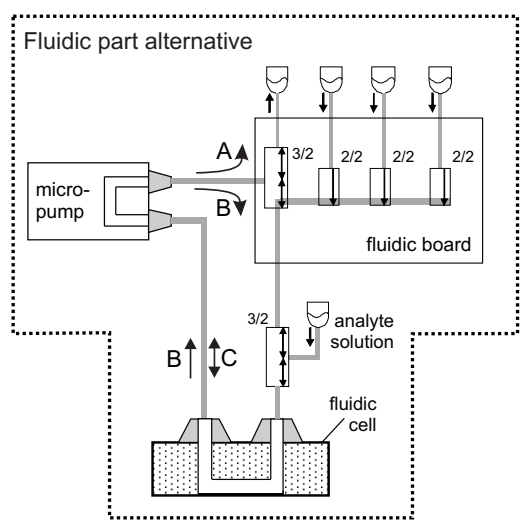

Fig. 11: Alternative fluidic setup: By pumping in alternating forward-backward flow direction $(C)$ compared to injection or cycling mode (A / B), as shown in Fig. 9 , smaller analyte solution volumes are necessary.

\subsection{Vibration}

The vibration of the sensor chip can strongly increase noise and drift during optical measurement. The main reason for this system is the displacement of the optical fibers, compared to the grating couplers. The vibration of the base plate, caused by the micropump and microvalves, was measured by using an accelerometer sensor giving a voltage proportional to the acceleration. The sensor was placed on a $15 \mathrm{~mm}$ thick aluminum base plate $\left(200 \times 100 \mathrm{~mm}^{2}\right)$ with a distance of $30 \mathrm{~mm}$ to the pump to study the vibration transmission to the system. The weight of the piezoelectric sensor was $1.8 \mathrm{~g}$ and the minimum resonance frequency $50 \mathrm{kHz}$. The output voltage signal was transformed by Fast Fourier Transformation (FFT) to determine the main vibration frequencies. The potential of influencing the amount of vibration on the sensor by damping was analyzed by using two exemplary absorber materials: a corrugated fiberboard (flute type $\mathrm{E}$, thickness $1.1 \mathrm{~mm}$ ) and a polyester foam sheet (high resilience, thickness $15 \mathrm{~mm}$, density $19 \mathrm{mg} \cdot \mathrm{cm}^{-3}$ ).

For the setup without a damping sheet, a high amount of vibration was guided to the base plate. The main vibrational frequencies were below $100 \mathrm{~Hz}$ (Fig. 12). The vibration intensity was strongly attenuated by the absorber materials. Especially with the foam sheet absorber, also the low-frequency vibrations could be minimized easily. This simple but effective possibility of damping, directly at the cause of vibration, promotes the later use of the micropump. However, pulses that travel inside and along the tubes as acoustic waves were not determined. This should be analyzed by testing the cross-sensitivity with the respective biosensor which will be a future task.

\subsection{Flow rates}

The flow rates of the test solutions should be defined and stable, to obtain reproducible experiments and stable sensor signals. The flow rates in this system were examined by using a flow rate device (IDA2Plus, BIO-TEK Instruments). Deionized water was used during measurement. Buffer, receptor and analyte solutions for biosensing experiments have similar viscosities like deionized water. Therefore, viscosity dependent measurements were not necessary. The flow rates of the micropump were about $7.5 \%$ higher than expected from the design, measured for $500 \mu l \cdot \mathrm{min}^{-1}$, but could easily be calibrated. After calibration, the flow rate standard deviation was $\pm 0.2 \%$ of the absolute value compared to $\pm 0.7 \%$ for a reference tubing pump (both at $\left.500 \mu \mathrm{l} \cdot \mathrm{min}^{-1}\right)$. The higher pulsation at low-frequency of the reference tubing pump arises as the main reason.

The device uses volumetric detection and measures one flow rate value every $5 \mathrm{~s}$ to $20 \mathrm{~s}$ depending on the absolute amount of the flow rate. Therefore, the high-frequency pulsation of the micropump could not be determined due to the applied measurement principle. This pulsation should be determined with a flow sensor, suited for these flow rates, and a resolution of at least $100 \mathrm{~Hz}$. 


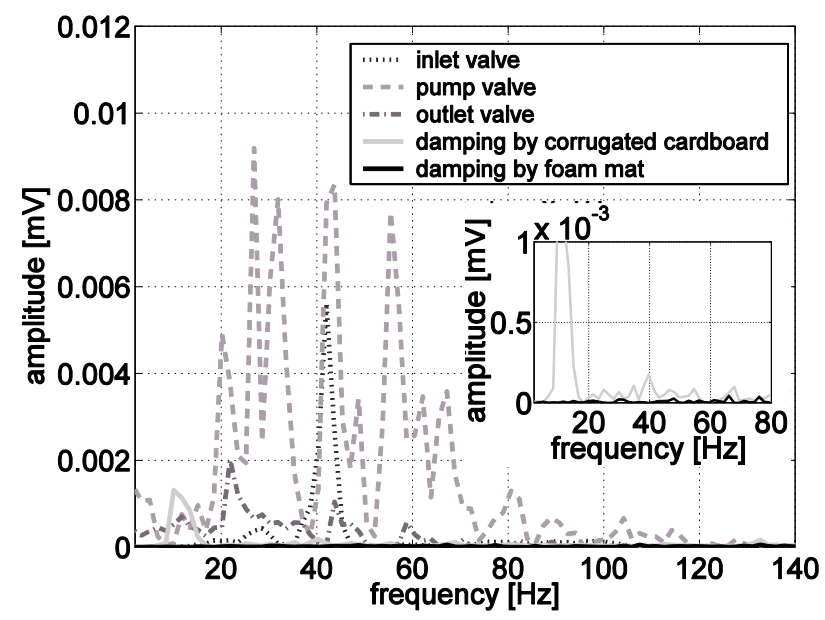

Fig. 12: Vibration spectrum of the base plate, caused by the micropump. The vibration could be strongly attenuated by simple absorber materials. The inset shows the vibration spectrum with absorber materials at a enlarged amplitude scale.

\subsection{Protein binding to the contact materials of the microfluidic system}

The fluidic system was developed for use in biosensing experiments, especially protein binding. All contact materials in the system have a low surface energy to minimize unwanted protein adsorption. Proteins should bind only to the biosensor surface. Binding to the fluidic contact materials reduces the protein concentration in the solution, and thus reduces sensing performance for fluidic modes $\mathrm{B}$ and $\mathrm{C}$ (Figs. 9, 11). Further, if cleaning after an experiment is insufficient, the bound protein can desorb in a following biosensor experiment and lead to contamination. The binding of proteins onto contact materials was analyzed using the model protein bovine serum albumin (BSA) which has a very high adsorption on most surfaces. It consists of about 600 amino acids and contains most chemical functional groups including $-\mathrm{COOH},-\mathrm{OH},-\mathrm{NH}_{2},-\mathrm{SH}$, which enables binding to most chemical groups. If BSA does not bind to an examined surface, this surface can be regarded as a low-sticking surface for most proteins. Therefore, BSA is best suited as a model protein for binding tests.

The materials PEEK and PTFE were cleaned with isopropanol and $\mathrm{N}_{2}$-dryed before used. ETFE was not examined since it has very similar polar and dispersive surface energies as PTFE. PDMS was used as manufactured. Functionalized polymethylmethacrylate (PMMA), which also serves as biosensor material, was utilized as a reference. For the functionalization of PMMA, hydroxyl groups were created by atmospheric pressure air plasma, followed by silanization with an aminosilane. BSA was used at a concentration of $1 \mathrm{mg} \cdot \mathrm{l}^{-1}$ in deionized water. It was either dispensed as a small droplet on the material surface or flowed over the surface at a flow rate of $250 \mu \mathrm{l} \cdot \mathrm{min}^{-1}$. A microfluidic channel with a crosssection of $0.09 \times 1 \mathrm{~mm}^{2}$ (height $x$ width) was used. The droplets remained for either $10 \mathrm{~min}$ or $30 \mathrm{~min}$. For the flow test, the same durations were used. The BSA binding was analyzed by fluorescence labeling with fluorescein isothiocyanate (FITC) and fluorescence microscopy. FITC was applied at a concentration of $0.1 \mathrm{mg} \cdot \mathrm{ml}^{-1}$ in a $\mathrm{Na}_{3} \mathrm{BO}_{3}$ buffer. Fig. 13 shows the BSA and FITC binding, schematically. The fluorescence intensity was analyzed with a fluorescence microscope taking 16 bit gray scale pictures as shown in Fig. 14. For the evaluation, only homogeneous areas with no residues (Fig. 14 (a)) were analyzed. The resulting diagrams (Figs. 15, 16) show average values of these homogeneous areas.

For fluorescence labeling, different methods for the analyses of protein binding to the fluidic materials were tested. No significant difference for the four different BSA treatments could be observed (Fig. 15). BSA binds quickly on the surfaces and the binding does not depend on the flow time. Because of the convenience of use and simplicity to distinguish BSA- / non-BSA-boundaries, the $30 \mathrm{~min}$ droplet method was chosen for further experiments. 


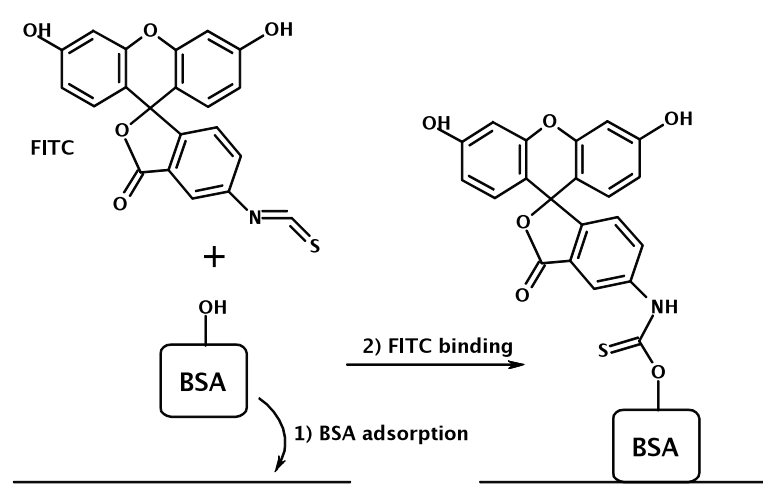

Fig. 13: Adsorption of the model protein BSA on the material surface and detection of the amount of adsorption by binding of the fluorophore fluorescein isothiocyanate (FITC).
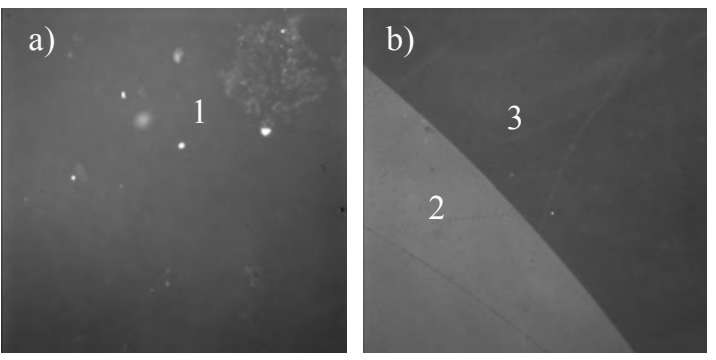

Fig. 14: Sample fluorescence pictures showing BSA untreated PTFE with some fluorescent residue (1) (a) and a BSA droplet treated PDMS (2) showing small fluorescence intensity compared to untreated PDMS (3) (b).

Fluorescence labeling generally showed only very small binding of BSA to the analyzed fluidic contact materials (Fig. 16) compared to functionalized PMMA which is used as a sensor material. Even though BSA tends to stick on surfaces easily, the tested fluidic contact materials should adsorb only small amounts of proteins during biosensing experiments. The binding to PEEK could not be analyzed, because of the high autofluorescence of the bulk material at the wavelengths used during fluorescence microscopy. For other fluorescence filter sets, the autofluorescence was still very high, so most other fluorescent labels cannot be analyzed as well. Ellipsometry may be applied here as an alternative method to determine the protein layer thicknesses.

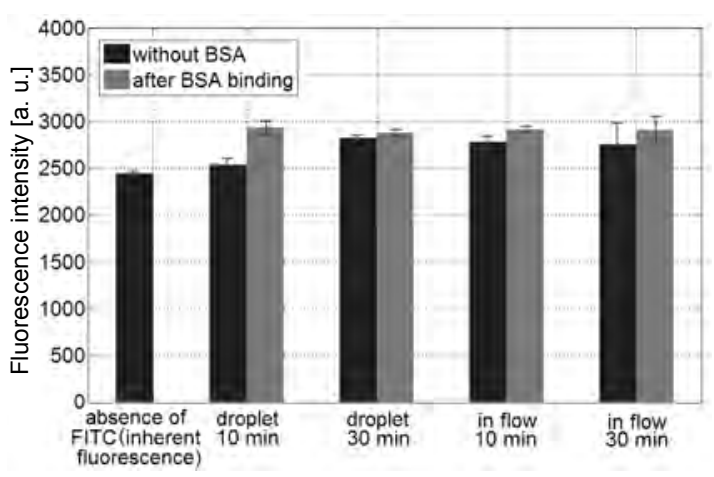

Fig. 15: Different methods for the analyses of protein binding to the fluidic materials, exemplarily shown for PDMS. A simple droplet method is sufficient and was used for further experiments $(30 \mathrm{~min}$ preferable due to slightly smaller standard deviations).

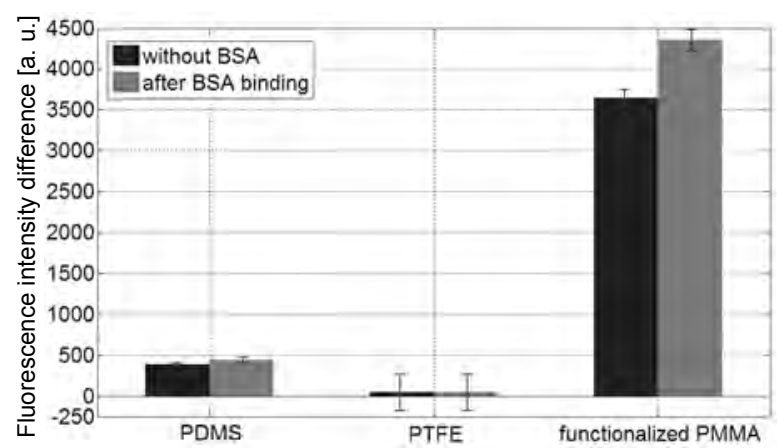

Fig. 16: Small binding of BSA to the fluidic contact materials (PDMS and PTFE/after BSA binding), compared to binding to the functionalized sensor material PMMA (funct. PMMA/after BSA binding). FITC also binds well directly to functionalized PMMA (funct. PMMA/without $B S A)$ due to the creation of $\mathrm{OH}$-groups after plasma treatment. All values represent the difference of the fluorescence after FITC-labeling to the autofluorescence of the respective material (without/with $B S A$ is both FITC-labeled).

\section{Conclusions}

The biosensor system proposed in this work is based on functional, polymeric microring resonators. It uses multilevel grating couplers and a microfluidic system for better automation, miniaturization and for a potential use for sensitive and fast POCT. The presented multilevel UV-NIL technologies and the optical grating couplers have a high potential for the mass production of cost-efficient and single-use biosensor chips. The presented microfluidic system is both miniaturized and robust, and allows the automation of entire biosensing experiments. The vibration of the micropump was analyzed and could be strongly minimized by simple absorber materials. The flow-rate was determined and could be calibrated. The influence of acoustic waves and high-frequent flow-rate pulses to the sensor chip should be determined in 
the final setup by carrying out further experiments. The adsorption and binding of the model protein BSA was analyzed to determine a potentially higher analyte consumption or later desorption and system contamination. The binding to the contact materials used in the fluidic system was much smaller than the binding to the functionalized sensor material. The proposed evaluation methods can be used to improve the design of fluidic systems during development.

\section{Acknowledgements}

This work was financed and scientifically supported by DFG Research Training Group DFG 1401/1 as well as the BMBF (FPP "Optical Biosensors"). The authors thank Alfred Kick and Martin Bönsch from Technische Universität Dresden, Department of Chemistry and Food Chemistry for their support with BSA binding, FITC labeling and valuable discussions. Thanks also to PD Dr. Uhlemann from the Technische Universität Dresden, Electronic Packaging Laboratory for his help with flow rate measurements.

\section{References}

[1] Fan, X.; White, I.; Shopova, S.; Zhu, H.; Suter, J. \& Sun, Y., "Sensitive optical biosensors for unlabeled targets: A review", analytica chimica acta, Elsevier, 2008, 620, 8-26.

[2] Yariv, A., "Critical coupling and its control in optical waveguide-ring resonator systems", Photonics Technology Letters, IEEE, IEEE, 2002, 14, 483-485.

[3] Washburn, A.; Luchansky, M.; Bowman, A. \& Bailey, R., "Quantitative, label-free detection of five protein biomarkers using multiplexed arrays of silicon photonic microring resonators", Analytical chemistry, ACS Publications, 2009, 82, 69-72.

[4] Chao, C. \& Guo, L., "Design and optimization of microring resonators in biochemical sensing applications", Journal of lightwave technology, IEEE, 2006, 24, 1395-1402.

[5] Hu, J.; Sun, X.; Agarwal, A. \& Kimerling, L., "Design guidelines for optical resonator biochemical sensors", JOSA B, Optical Society of America, 2009, 26, 1032-1041.

[6] Chao, C. \& Guo, L., "Reduction of surface scattering loss in polymer microrings using thermal-reflow technique", Photonics Technology Letters, IEEE, IEEE, 2004, 16, 1498-1500.

[7] Luchansky, M. et al., "Characterization of the evanescent field profile and bound mass sensitivity of a labelfree silicon photonic microring resonator biosensing platform", Biosensors and Bioelectronics, Elsevier, 2010, 26, 1283-1291.

[8] Armani, A.; Kulkarni, R.; Fraser, S.; Flagan, R. \& Vahala, K., "Label-free, single-molecule detection with optical microcavities", Science, American Association for the Advancement of Science, 2007, 317, 783.

[9] Chao, C.; Fung, W. \& Guo, L., "Polymer microring resonators for biochemical sensing applications", Selected Topics in Quantum Electronics, IEEE Journal of, IEEE, 2006, 12 (1), 134-142.

[10] Kirchner, R., "On UV-Nanoimprint-Lithography as Direct Patterning Tool for Polymeric Microsystems", PhD Thesis, Technische Universität Dresden, 2012.

[11] Ofir, Y.; Moran, I.; Subramani, C.; Carter, K. \& Rotello, V., "Nanoimprint Lithography for Functional ThreeDimensional Patterns", Advanced Materials, Wiley Online Library, 2010, 22, 3608-3614.

[12] Kirchner, R.; Kaiser, M.; Adolphi, B.; Landgraf, R. \& Fischer, W., "Chemical functional polymers for direct UV assisted nanoimprinting of polymeric photonic microring resonators", Physica Status Solidi A, 2011, 208, 13081314.

[13] Roelkens, G.; Vermeulen, D.; Van Laere, F.; Selvaraja, S.; Scheerlinck, S.; Taillaert, D.; Bogaerts, W.; Dumon, P.; Van Thourhout, D. \& Baets, R., "Bridging the gap between nanophotonic waveguide circuits and single mode optical fibers using diffractive grating structures", Journal of Nanoscience and Nanotechnology, American Scientific Publishers, 2010, 10, 1551-1562.

[14] TIRF Technologies. Online at www.tirftechnologies.com, accessed at Jan. 20, 2012.

[15] Gesim Microfluidics. Online at www.gesim.de, accessed at Jan. 20, 2012.

[16] Syrris Flow Products. Online at syrris.com, accessed at Jan. 20, 2012.

[17] Landgraf, R.; Kaiser, M.; Posseckardt, J.; Adolphi, B. \& Fischer, W., "Functionalization of Polymer Sensor Surfaces by Oxygen Plasma Treatment", Procedia Chemistry, Elsevier, 2009, 1, 1015-1018.

[18] Landgraf, R.; Haugwitz, T.; Kirchner, R.; Finn, A. \& Fischer, W., "Planar Optical Waveguide Design for UVNanoimprinted Microring Resonator Based Biosensors", 10th IEEE Sensors Conference, 2011, 596 - 599.

[19] Finn, A.; Jahn, A.; Kirchner, R.; Künzelmann, U.; He, J.; Waegner, M. \& Fischer, W., "Multilayer Nano-ImprintLithography Mold Fabrication Process", GMM Workshop Mikro-Nano-Integration / GMM-Fachbericht 68, 2011, 8-12. 